\title{
THE SOFT X-RAY BLAST IN THE APPARENTLY SUBLUMINOUS GRB 031203
}

\author{
D. Watson, ${ }^{1}$ S. A. Vaughan, ${ }^{2}$ R. Willingale, ${ }^{2}$ J. Huorth, ${ }^{1}$ S. Foley, ${ }^{3}$ J. P. U. Fynbo, ${ }^{1}$ P. Jakobsson, ${ }^{1}$ A. Levan, ${ }^{2}$ \\ P. T. O’Brien, ${ }^{2}$ J. P. Osborne, ${ }^{2}$ K. Pedersen, ${ }^{1}$ J. N. Reeves, ${ }^{4,5}$ J. A. Tedds, ${ }^{2}$ and M. G. Watson ${ }^{2}$ \\ Received 2005 July 5; accepted 2005 September 15
}

\begin{abstract}
GRB 031203 was a very low apparent luminosity $\gamma$-ray burst (GRB). Coincidentally, it was also the first GRB with a dust-scattered X-ray halo. The observation of the halo allowed us to infer the presence of a large soft X-ray fluence in the total burst output. It has also been claimed, however, that GRB 031203 was intrinsically subenergetic, representative of a class of spectrally hard, low-energy bursts quite different from other GRBs. A careful reanalysis of the available data confirms our original finding that GRB 031203 had a very large soft X-ray component, the time of which can be constrained to within a few minutes after the burst, strongly suggesting that while GRB 031203 did indeed have a very low apparent luminosity, it was also very soft. Notions propagated in the literature regarding the uncertainties in the determination of the soft X-ray fluence from the halo data and on the available constraints from the hard X-ray data are addressed: the properties of the scattering dust along the line of sight (grain sizes, precise location, and geometry) are determined directly from the high-quality X-ray data so that there is little uncertainty about the scatterer; constraints on the X-ray light curve from the INTEGRAL spacecraft at the time of the soft X-ray blast are not complete because of a slew in the spacecraft pointing shortly after the burst. Claims that GRB 031203 was intrinsically underenergetic and that it represents a deviation from the luminosity-peak-energy relation do not appear to be substantiated by the data, regardless of whether the soft X-ray component is (arbitrarily) declared part of the prompt emission or the afterglow. We conclude that the difference between the soft and hard X-ray spectra from XMM-Newton and INTEGRAL indicate that a second soft pulse probably occurred in this burst, as has been observed in other GRBs, notably GRB 050502B.
\end{abstract}

Subject headings: gamma rays: bursts — X-rays: general — X-rays: ISM

\section{INTRODUCTION}

While $\gamma$-ray bursts (GRBs) are no longer as enigmatic as they were even a few years ago, the ability to use GRBs as a serious tool in cosmology and an understanding of their basic mechanisms still elude us. Relations based on the energy release have the potential to resolve these difficulties.

In particular, the "Amati relation" (Amati et al. 2002) between the equivalent isotropic $\gamma$-ray total energy $\left(E_{\text {iso }}\right)$ and the spectral peak energy $E_{\text {peak }}$ in GRBs has been the focus of considerable recent work (e.g., Band \& Preece 2005; Nakar \& Piran 2005; Ghirlanda et al. 2004). Only a single burst, apart from GRB 031203 , has extended this relation to very low luminosities and peak energies (i.e., the low-luminosity XRF 020903; Sakamoto et al. 2004).

It has also been suggested that the total energy in $\gamma$-rays from a GRB is nearly constant at $\sim 10^{51}$ ergs (Frail et al. 2001), by correcting for the opening angle of the putative GRB jet. The determination of the opening angle is dependent on the time of the break in the light curve. This measure has proved difficult to use or understand because of (1) the difficulty in deciding the

\footnotetext{
1 Dark Cosmology Centre, Niels Bohr Institute, University of Copenhagen, Juliane Maries Vej 30, DK-2100 Copenhagen Ø, Denmark; darach@astro.ku.dk, jens@astro.ku.dk, pallja@astro.ku.dk, kp@astro.ku.dk.

2 X-Ray Astronomy Group, Department of Physics and Astronomy, Leicester University, Leicester LE1 7RH, UK; anl@star.le.ac.uk, pto@star.le.ac.uk,julo@ star.le.ac.uk, jat@star.le.ac.uk, sav2@star.le.ac.uk,mjw@star.le.ac.uk,rw@star le.ac.uk.

3 Department of Experimental Physics, University College Dublin, Belfield, Dublin 4, Ireland; sfoley@bermuda.ucd.ie.

${ }^{4}$ Laboratory for High Energy Astrophysics, Code 662, NASA Goddard Space Flight Center, Greenbelt, MD 20771; jnr@milkyway.gsfc.nasa.gov.

5 Universities Space Research Association.
}

jet break time in light curves that are often sparsely sampled, contaminated by supernova features, and subject to fluctuations caused by density variations, and (2) the (few) cases where the total apparent energy release (equivalent isotropic) is well below this value.

By any measure, the apparent isotropic energy output in GRB 031203 was extremely low (Watson et al. 2004, hereafter W04), and for any opening angle of the jet, was significantly below the standard energy of $\sim 10^{51}$ ergs for GRBs inferred from jet opening angles (W04). Sazonov et al. (2004, hereafter SLS04) find an isotropic equivalent energy release of $4 \pm 1 \times 10^{49} \mathrm{ergs}$ from the International Gamma-Ray Astrophysics Laboratory (INTEGRAL) $20-200 \mathrm{keV}$ spectrum (an observed fluence of $2.0 \pm 0.4 \times$ $10^{-6} \mathrm{ergs} \mathrm{cm}^{-2}$ ). Other bursts (e.g., XRF 020903) also have apparent energies below $\sim 10^{51}$ ergs.

It has been argued by SLS04 and Soderberg et al. (2004, hereafter S04) that GRB 031203 was representative of a new class of intrinsically subenergetic bursts, possessing many of the characteristics of classical GRBs, but being a thousand times less powerful. This claim has far-reaching implications for GRBs. Ambitions to use GRBs as the most powerful distance indicators in cosmology currently seem to lie mostly with the $E_{\text {peak }}-E_{\gamma}$ relation (similar to the Amati relation, but using the total collimationcorrected $\gamma$-ray energy release, $E_{\gamma}$; Ghirlanda et al. 2004), but whatever relation is used, a low-redshift calibration sample will be essential. If there is a distinct population of underenergetic bursts, it will clearly need to be well described and calibrated differently, especially if this type of burst dominates the lowredshift sample.

To suggest that GRB 031203 was intrinsically subenergetic and a member of a new class of such bursts, we must answer the question: was the total burst energy of GRB 031203 lower than 
expected compared to other GRBs? Such an apparently faint burst is expected to be soft, according to the Amati relation. Under the assumption that the emission detected by INTEGRAL comprised the entire burst, GRB 031203 was indeed much fainter than expected from this relation, since the INTEGRAL spectrum is hard. The high value of $E_{\text {peak }}$ adopted $(>190 \mathrm{keV})$ was based on the hard X-ray spectrum of the single pulse detected by the INTEGRAL satellite. But as we have shown (W04 and Vaughan et al. 2004, hereafter V04), the transient dust-scattered X-ray halo associated with the burst indicates that it was also very rich in soft $\mathrm{X}$-rays; otherwise, the halo observed by $X M M$-Newton could not have been so bright.

The argument that GRB 031203 was a member (with GRB 980425) of a new, intrinsically underenergetic class of GRBs (SLS04; S04) hinges on the hardness of the burst.

The fluence in the soft X-ray blast is critical to this discussion.

The $X M M$-Newton data are therefore carefully reanalyzed in this paper. The dominant uncertainties in deriving the fluence are outlined in $\S 2$. The full spectrum of GRB 031203 and the consequences of analyzing the complete data set are presented and discussed in $\S 4$. A cosmology with $\Omega_{m}=0.3, \Omega_{\Lambda}=0.7$ and $H_{0}=75 \mathrm{~km} \mathrm{~s}^{-1} \mathrm{Mpc}^{-1}$ is assumed throughout. Error ranges quoted are $90 \%$ confidence intervals, unless stated otherwise.

\section{METHOD AND UNCERTAINTIES}

Details of the XMM-Newton observations and the initial data analyses for GRB 031203 are outlined in V04 and W04. The luminosity of the soft X-ray blast, inferred from the dust-scattered halo observed by XMM-Newton, is key to the nature of GRB 031203. Here, we outline the procedure used to derive the fluence and analyze the major sources of uncertainty in this calculation.

A complete model of the X-ray halo was used to find the bestfit parameters, including the rate of expansion, width, total fluence, and flux decay of the rings. The model produces a two-dimensional distribution for the halo brightness with time and scattering angle for a given energy band.

The fluence of the X-ray blast was inferred from the observed halo fluence divided by the scattering fraction. The differential scattering fraction as a function of scattering angle at a given energy is found by integrating scattering cross sections over the dust grain size $(a)$ distribution up to the maximum grain size $\left(a_{\max }\right)$ and multiplying by the column density of dust. The uncertainty in the inferred blast fluence largely reflects the uncertainties in the scattering dust, which are dominated by two things: (1) the size of the scattering dust column, and (2) the dust grain size distribution.

\subsection{The Scattering Dust Column}

It was argued by Prochaska et al. (2004) and later by SLS04 that the fluence derived from the X-ray halo could have been overestimated by a factor of 4.4 in our previous work (V04). This was based on two incorrect assumptions.

The first was that the individual rings observed in the halo were scattered by the total dust column along the line of sight $\left(A_{V}=3.6\right){ }^{6}$ whereas in fact the dust contributing to the rings is confined to relatively thin sheets of dust at well-defined distances $^{7} 1395_{-30}^{+15}$ and $868_{-16}^{+17} \mathrm{pc}$ (see below). Dust that is not

\footnotetext{
${ }^{6}$ A higher $A_{V}$ means a larger fraction of the X-rays are scattered, which in turn would imply a smaller "blast" fluence for a given observed fluence in the halo.

7 The distance to the scatterer is known from fitting the halo's angular expansion with time; from geometrical arguments, $D=2 c \tau / \theta^{2}$, where $D$ is the distance to the scatterer, $\tau$ is the delay between arrival times of directly observed and scattered photons, and $\theta$ is the observed angle (see V04 for more details).
}

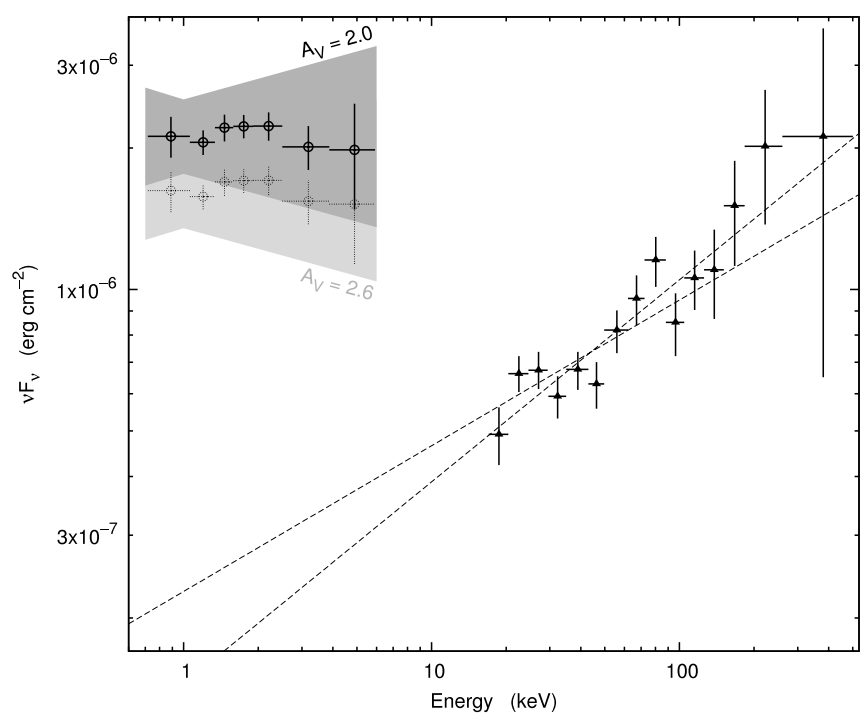

FIG. 1.- Spectral energy distribution of the pulses detected using the dustscattered X-ray halo and directly with INTEGRAL. The data from the X-ray halo are plotted as open circles, with the uncertainty in the correction for the dust scattering plotted as a gray butterfly. The closed triangles represent data from the direct observation by INTEGRAL's IBIS instrument (SLS04), with the $90 \%$ limits to the best-fitting power law ( photon index, $\Gamma=1.63 \pm 0.06$ ) plotted as dashed lines; the fluence at $1 \mathrm{keV}$ derived from the halo cannot be made consistent with it.

contained in these sheets cannot contribute to the scattered rings, and since we use only the X-ray fluence in the rings themselves to derive the total fluence, other dust along the line of sight is irrelevant to the calculation of the burst fluence. Even using $A_{V}=3.6$ as the extreme upper limit to the dust column contained in the sheets does not change our results by more than a factor of 1.8. At the same time, it was also argued by Prochaska et al. (2004) that $A_{V} \sim 1$ of the total dust column actually belongs to the GRB host galaxy, which would leave only $A_{V} \sim 2.6$ as the upper limit to the dust column available for the Galactic dust sheets. We find it unlikely that the entire dust column in this direction is contained in these two sheets. Based on the Galactic radial dust profiles (Neckel et al. 1980), the most likely value in the sheets is in fact $A_{V} \sim 2.0$ (V04).

The second misapprehension was that the dust scattering fraction scales exponentially with $A_{V}$, whereas the dependence scales with the optical depth and is therefore only linearly related to the column density (Mauche $\&$ Gorenstein 1986). The factor 4.4 is the difference in optical extinction between $A_{V}=3.6$ and $A_{V}=2.0$, not the column density. This extinction relation is not correct for the X-ray scattering where the relationship is essentially linear. Since the maximum $A_{V}$ has been argued to be $\sim 2.6$, the fluence in the burst could only have been overestimated by at most 2.6/2.0, which is $\sim 30 \%$, though as noted above, this is unlikely. The effect of using $A_{V}=2.6$ to derive the X-ray burst fluence is illustrated by dotted open circles and a lighter gray butterfly in Figure 1.

\subsection{The Grain Size Distribution}

Because we possess time-resolved data for the evolution of the X-ray halo, the shape of the angular scattering response function for the dust (i.e., the way the scattered flux falls off with the scattering angle, Fig. 2) is strongly constrained. The largest grains always dictate this angular scattering response function, allowing us to fit the differential scattering cross sections to the observed flux in the halo as a function of scattering angle, with $a_{\max }$ as a 


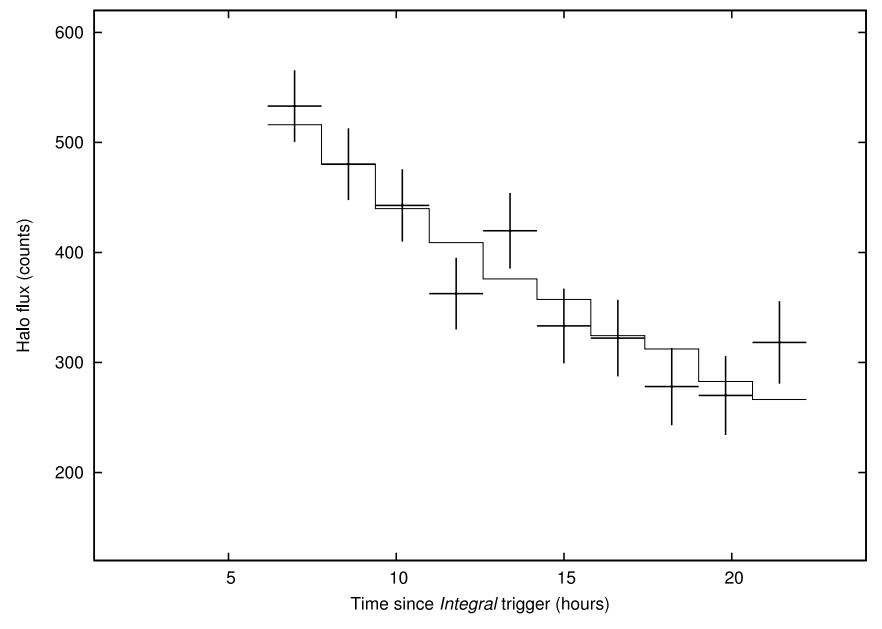

FIG. 2.-Flux decay of the soft X-ray halo with time, with best-fit model based on the dust-grain angular scattering profile. Over the period of the observation, the total flux in the scattered halo decreases as the scattering from increasingly large angles is observed. Increasing the grain sizes makes this decay rate faster because larger grains scatter more efficiently at smaller angles. The maximum of the grain size distribution $\left(a_{\max }\right)$ dominates the scattering profile. We can therefore fit to the measured decay of the halo to find $a_{\max }$. In this case, $a_{\max }=0.50 \pm 0.03 \mu \mathrm{m}$.

free parameter. This allows us to say that $a_{\max }=0.50 \pm 0.03 \mu \mathrm{m}$ along this line of sight.

In our original analysis (V04), a single grain size was used. Here we assume a distribution proportional to $a^{-3.5}$, which gives a good representation of the optical extinction curve and is similar to that observed in X-ray scattering for Galactic sources (Mathis et al. 1977; Mauche \& Gorenstein 1986; Predehl \& Schmitt 1995).

Running the model with different values of the power-law index of the grain size distribution, it is clear that values below -4.0 yield very large total scattering fractions per $A_{V}(>12 \%)$, well above any observed value (Draine 2003). Even using the steep value of -4.0 implies a fluence only $\sim 33 \%$ smaller than our best estimate.

The results from this analysis are consistent with our previous estimate (V04), which used a single grain size, based on the dust-scattering efficiencies found for Galactic X-ray halo sources (Predehl \& Schmitt 1995). In other words, a similar Galactic source halo would have close to the same brightness for its central source as we infer for GRB 031203.

The scattering efficiency is not very sensitive to variations in the details of the physical grain model.

\subsubsection{Dust Scattering Efficiency}

The dust model of Weingartner \& Draine (2001), which has been used (e.g., Draine \& Bond 2004) to convert optical extinction $\left(A_{V}\right)$ to $\mathrm{X}$-ray scattering factor $\left(\tau_{\text {sca }}\right)$, gives a scattering factor that is consistently $2-4$ times larger, over the $0.7-3 \mathrm{keV}$ range, than empirically determined from Galactic sources using the Advanced Satellite for Cosmology and Astrophysics (ASCA), Röntgensatellit (ROSAT), and Chandra (Woo et al. 1994; Predehl \& Schmitt 1995; Smith et al. 2002; Yao et al. 2003). Using this model, the fluence in the X-ray blast would be lower by a factor of $\sim 3$. However, as this model does not agree with the observational comparison of $\tau_{\text {sca }}$ and $A_{V}$ (see Fig. 11 of Draine 2003), we have continued to use the empirically determined value from Predehl \& Schmitt (1995). It is worth noting that the $\tau_{\text {sca }}-A_{V}$ relation is strongly dependent on the grain size distribution (see above), so that these are not independent sources of uncertainty.

\section{RESULTS}

Our reanalysis results in a $1 \mathrm{keV}$ fluence density of $1320 \pm$

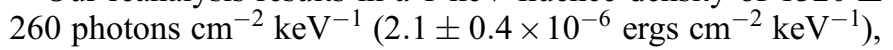
a factor of 10 above an extrapolation of the INTEGRAL powerlaw spectrum to $1 \mathrm{keV}$ (which has a $1 \mathrm{keV}$ fluence density of $110 \pm 20$ photons $\mathrm{cm}^{-2} \mathrm{keV}^{-1}$ and a photon index $\Gamma=1.63 \pm$ 0.06; Fig. 1). The uncertainties quoted for the X-ray halo data include the measurement error and uncertainties related to the halo modeling. Given the size of these uncertainties and the fact that they are based on direct observation along this line of sight, we are forced to conclude that it is unlikely that the $1 \mathrm{keV}$ fluence of the blast could have been substantially different.

The analysis of the halo expansion was also improved by allowing the time of the X-ray blast to be a free-fit parameter; Gaussian profiles were fit to the halo at different times to improve the radial size estimates, and the model fit was integrated over each time bin. We find results consistent with our previous work. The time of the blast was $600 \pm 700 \mathrm{~s}$ after the beginning of the burst detected by INTEGRAL. The distances to the scatterers of $1395_{-30}^{+15}$ and $868_{-16}^{+17} \mathrm{pc}$ are some of the most accurately known distances to any object beyond about 50 pc, with a total uncertainty of only $\lesssim 2 \%$ at $\sim 1 \mathrm{kpc}$.

\section{DISCUSSION}

The peculiar spectral energy distribution (SED) of the complete data set points to the fact that INTEGRAL and XMM-Newton observed different events in GRB 031203. A natural interpretation of these data is that there were two pulses in GRB 031203: one detected by INTEGRAL, with a hard spectrum peaking at or above $\sim 190 \mathrm{keV}$, and a second pulse with a much softer spectrum, detected by XMM-Newton via its dust-scattered halo.

It is expected that INTEGRAL's IBIS instrument should, in its lowest energy channels, have detected the harder X-rays associated with such a powerful soft X-ray blast (Sazonov 2004, private communication). However, the light curve limits obtained by INTEGRAL are incomplete. Long ( $\$ 40 \mathrm{~s}$ ) data gaps exist due to a $\sim 100 \mathrm{~s}$ slew of the satellite. The slew occurred less than $300 \mathrm{~s}$ after the initial pulse. The IBIS data cannot therefore be used to place useful limits on the soft flux in the burst. (It may, however, be used to place limits on the timing of the X-ray blast).

Many bursts exhibit multiple pulses often accompanied by a strong softening of the spectrum, e.g., GRB 960720, GRB 970228 (Frontera et al. 2000), or GRB 020410 (Nicastro et al. 2004). The most striking case so far appeared during the preparation of this paper; the detection of a massive soft X-ray flare in GRB 050502B (Burrows et al. 2005) starting $\sim 500 \mathrm{~s}$ after the initial $\gamma$-ray pulse and lasting $\sim 500 \mathrm{~s}$. The fluence in the soft X-ray flare was comparable to the first $\gamma$-ray pulse, which had a hard spectrum. (Indeed, the photon spectral index, $\Gamma=1.6$, was very similar to that observed in the $\gamma$-ray pulse of GRB 031203.) The consistency between the features observed in GRB 050502B and those inferred here from the X-ray halo of GRB 031203 reinforces the interpretation that there were two very different pulses in GRB 031203.

\subsection{Afterglow or Prompt Emission?}

The complete data show that although GRB 031203 was very faint (W04), there is no reason to suppose that it was anomalously so; it is more luminous than XRF 020903, for instance, and probably of comparable luminosity with XRF 030723 (Fynbo et al. 2004; Tominaga et al. 2004). The key issue is therefore whether it deviates significantly from the Amati relation, i.e., whether it is spectrally hard. The interpretation of the event as prompt emission, 
highly unusual afterglow, or reverse shock, while interesting speculation (SLS04), is not relevant to whether or not the burst was unexpectedly faint. The comparison is an observational one; i.e., Amati et al. (2002) used the emission detected by the BeppoSAX burst monitor and Wide Field Camera (WFC). To compare with these bursts in a meaningful way, we must use the same observational criteria and must include the soft X-ray blast in the calculation of the total luminosity, since its fluence or minimum flux would have been detected by the WFC (Amati et al. 2002). The consideration of whether certain parts of the emission should or could be considered as afterglow or prompt emission is irrelevant for this comparison, which is an observational one, based on the criteria for the sample selection. Based purely on the INTEGRAL data, GRB 031203 appears to be one of only two significant outliers from this relation (the other being GRB 980425). However, when we include the XMM-Newton data, the X-ray $(2-30 \mathrm{keV})$ to $\gamma$-ray $(30-400 \mathrm{keV})$ fluence ratio is $S_{\mathrm{X}} / S_{\gamma}=1.8_{-0.9}^{+0.4}$, which indicates that GRB 031203 was probably an X-ray flash, and certainly at least X-ray rich. This implies not only that the lower bound to the total X-and $\gamma$-ray fluence in the burst was roughly twice the $2 \times 10^{-6}$ given by SLS04, but more importantly, that the peak energy of the total burst (if this is a well-defined concept in this case) was likely very low, as originally concluded in W04. Taking this into account, we conclude that there is no compelling evidence in the GRB energetics to suggest that GRB 031203 was intrinsically underenergetic.

In support of the argument that GRB 031203 was a cosmic analogue of GRB 980425, it was suggested that the shape of the prompt emission was the same in both bursts (single pulse and fast rise, exponential decay [FRED] shape; S04). Since GRB 031203 could certainly have possessed multiple pulses, this suggestion is not compelling.

The luminosity of the X-ray afterglow at about 1 day is also very faint $\left(9 \times 10^{42} \mathrm{ergs} \mathrm{s}^{-1}\right.$ at $10 \mathrm{hr}$; W04 $)$. The X-ray afterglow is, however, still 2 orders of magnitude (Ramirez-Ruiz et al. 2005) brighter than predicted in the subenergetic model proposed by $\mathrm{S} 04$; both this and the low energy inferred from radio calorimetry $\left(1.7 \times 10^{49}\right.$ ergs; S04) can readily be explained in a standard energy, off-axis model (Ramirez-Ruiz et al. 2005), which suggests an intrinsic peak energy for the burst of a few hundred keV, 1 order of magnitude above the total observed XMM-Newton+INTEGRAL value.

We acknowledge benefits from collaboration within the EU FP5 Research Training Network, "Gamma-Ray Bursts: An Enigma and a Tool." This work was also supported by the Danish Natural Science Research Council (SNF).
Amati, L., et al. 2002, A\&A, 390, 81

Band, D. L., \& Preece, R. D. 2005, ApJ, 627, 319

Burrows, D. N., et al. 2005, Science, 309, 1833

Draine, B. T. 2003, ApJ, 598, 1026

Draine, B. T., \& Bond, N. A. 2004, ApJ, 617, 987

Frail, D. A., et al. 2001, ApJ, 562, L55

Frontera, F., et al. 2000, ApJS, 127, 59

Fynbo, J. P. U., et al. 2004, ApJ, 609, 962

Ghirlanda, G., Ghisellini, G., \& Lazzati, D. 2004, ApJ, 616, 331

Mathis, J. S., Rumpl, W., \& Nordsieck, K. H. 1977, ApJ, 217, 425

Mauche, C. W., \& Gorenstein, P. 1986, ApJ, 302, 371

Nakar, E., \& Piran, T. 2005, MNRAS, 360, L73

Neckel, T., Klare, G., \& Sarcander, M. 1980, A\&AS, 42, 251

Nicastro, L., et al. 2004, A\&A, 427, 445

Predehl, P., \& Schmitt, J. H. M. M. 1995, A\&A, 293, 889

Prochaska, J. X., et al. 2004, ApJ, 611, 200

\section{REFERENCES}

Ramirez-Ruiz, E., Granot, J., Kouveliotou, C., Woosley, S. E., Patel, S. K., \& Mazzali, P. A. 2005, ApJ, 625, L91

Sakamoto, T., et al. 2004, ApJ, 602, 875

Sazonov, S. Y., Lutovinov, A. A., \& Sunyaev, R. A. 2004, Nature, 430, 646 (SLS04)

Smith, R. K., Edgar, R. J., \& Shafer, R. A. 2002, ApJ, 581, 562

Soderberg, A. M., et al. 2004, Nature, 430, 648 (S04)

Tominaga, N., Deng, J., Mazzali, P. A., Maeda, K., Nomoto, K., Pian, E., Hjorth, J., \& Fynbo, J. P. U. 2004, ApJ, 612, L105

Vaughan, S., et al. 2004, ApJ, 603, L5 (V04)

Watson, D., et al. 2004, ApJ, 605, L101 (W04)

Weingartner, J. C., \& Draine, B. T. 2001, ApJ, 548, 296

Woo, J. W., Clark, G. W., Day, C. S. R., Nagase, F., \& Takeshima, T. 1994, ApJ, 436, L5

Yao, Y., Zhang, S. N., Zhang, X., \& Feng, Y. 2003, ApJ, 594, L43 